\title{
Felix Syrovatka
}

\section{Nuit Debout: Frankreich gerät in Bewegung!}

Am frühen Morgen des 11. April 2016 räumte die Polizei die Place de la République im Herzen von Paris und zerstörte Zelte und Infrastruktur der noch jungen französischen Bewegung Nuit Debout („Die Nacht erhebt sich“). Doch schon am selben Abend protestierten wieder Tausende gegen die Arbeitsmarktpolitik der sozialistischen Regierung. Ausgangspunkt waren die Demonstrationen Ende März, an deren Anschluss mehrere hundert Menschen die Place de la République besetzten und anfingen, eine Infrastruktur aufzubauen. Vernetzt über die sozialen Medien, wie Facebook und Twitter, breiteten sich die Platzbesetzungen in mehr als 60 französischen Städten, darunter allen großen, aus. Selbst in traditionell eher konservativeren Städten wie Nizza existieren Ableger der Nuit-Debout-Bewegung.

Anfang der 1990er Jahre überschritt die Arbeitslosenquote die Zehn-ProzentMarke und zementierte die Arbeitslosigkeit als drängendstes Problem. Die steigende Arbeitslosigkeit war das Ergebnis der seit der angebotspolitischen Wende des damaligen Präsidenten François Mitterand 1984 eingeleiteten Transformation des französischen Kapitalismusmodells. Das Ende des Colbertismus und der 'Planification', die Deregulierungen im Finanzsektor sowie Privatisierungen hatten enorme Folgen für die soziale und territoriale Entwicklung des Landes. So weist etwa der ehemals industrialisierte Norden des Landes seit Anfang der 1990er Jahre eine konstant hohe Arbeitslosigkeit auf, die gleichzeitig mit einer Verschärfung der räumlichen Ungleichheit beim Zugang zu öffentlichen Dienstleistungen verbunden ist. Ebenso kam es, wie Boltanski und Chiapello (2006) darlegen, seit Ende der 1980er Jahre zu einem radikalen Wandel der Arbeitsverhältnisse, der mit einem kontinuierlichen Anstieg von befristeten Arbeitsverhältnissen und Leiharbeit verbunden war. Zwischen 1985 und 2001 stieg allein die Anzahl der durch Leiharbeit geleisteten Stunden um 506 Prozent (Delbar/Léonard 2002: 6). Im Jahr 2014 lag der Anteil befristeter Arbeitsverträge bei Neueinstellungen bei 84 Prozent, der höchste Wert in der gesamten EU. Zugleich ist der Arbeitsmarkt heute durch eine hohe strukturelle Arbeitslosigkeit geprägt und stark fragmentiert. Junge Menschen, Frauen oder MigrantInnen werden durch dieses System der prekären Beschäftigung diskriminiert und vom Arbeitsmarkt ausgeschlossen, womit sie in besonderem Maße von Arbeitslosigkeit betroffen sind (Lux 2015). So lag die Jugendarbeitslosigkeit im Februar 2016 mit 24,7 Prozent auf einem neuen Rekordhoch.

Die konstant hohe Arbeitslosigkeit führte dazu, dass die französische Arbeitsmarkt- und Beschäftigungspolitik in den letzten drei Jahrzehnten durch einen erhöhten und permanent aufrechterhaltenen Reformdruck gekennzeichnet war. Vor allem ab Ende der 1980er Jahre kam es auf der Grundlage der diskursiven 
Verknüpfung von Lohnnebenkosten und Arbeitslosigkeit zu einer Erneuerung der Arbeitsmarktpolitik und einer Welle von Reformen, die bis heute eine Kontinuität darstellen (vgl. Syrovatka 2016). So zielten fast alle Arbeitsmarkreformen seit den 1990er Jahren strukturell auf eine Abgabenentlastung der Arbeitgeber, eine Deregulierung des Arbeitsmarktes, auf die Stärkung der betrieblichen Ebene und eine stärkere Aktivierung von Arbeitslosen. Die einzige Ausnahme stellte die Arbeitsmarktpolitik der sozialistischen Regierung Jospin dar. Diese verfolgte eine nachfrageorientierte Beschäftigungspolitik und setzte 1998 die 35-Stunden-Woche gegen den vereinten Widerstand der Arbeitgeber durch, die seitdem jedoch Ziel ihrer Angriffe und denen konservativer Regierungen ist. Schon die nachfolgende Regierung Raffrain begann mit einer partiellen Rücknahme der 35-Stunden-Woche (Vail 2010).

Mit der Transformation der Finanzkrise in eine europäische Wirtschafts- und Staatsschuldenkrise wuchs der Druck vonseiten der EU und der OECD auf die französische Regierung stark an (Syrovatka 2016). Während François Hollande 2012 mit dem Versprechen gewählt wurde, die europäische Austeritätspolitik zu beenden und für mehr soziale Gerechtigkeit in Frankreich zu sorgen, schwenkte er im Januar 2014 ebenfalls auf einen angebotspolitischen Kurs ein, vorbereitet durch einen alarmierenden Bericht des ehemaligen EADS-Vorsitzenden Louis Gallois ${ }^{1}$ im

1 Der Bericht stellte die bisherige Wirtschaftspolitik der Regierung infrage. Basierend auf dem Narrativ fehlender Wettbewerbsfähigkeit, forderte er eine drastische Reduzierung der Lohnnebenkosten und einen "choc de compétitivite" durch Unternehmensentlastungen.
November 2012 zur Wettbewerbsfähigkeit Frankreichs. Noch im Dezember 2012 beschloss die sozialistische Regierung einen sogenannten Wettbewerbspakt, der Steuererleichterungen für Unternehmen von mehr als 20 Milliarden Euro im Jahr beinhaltete. Dem schloss sich Anfang 2013 die Rahmenvereinbarung der Sozialpartner über die „Flexicurity“ an, die Gehalts- und Arbeitszeitregelungen auf der betrieblichen Ebene flexibilisierte und Entlassungen aus wirtschaftlichen Gründen erleichterte. Die Arbeitsmarktpolitik von Hollande folgte damit schon vor der Neujahrsansprache 2014 einer angebotspolitischen Stoßrichtung. Die endgültige neoliberale Wende Hollandes vollzog sich dann mit der Durchsetzung des Verantwortungspaktes (PdR) und manifestierte sich in der Umbildung der Regierung, mitsamt der Berufung Manuel Valls zum Premierminister. Der PdR, der weitere Steuer- und Abgabenentlastungen für Unternehmen von mehr als 30 Milliarden Euro jährlich vorsieht, war der Startschuss einer ganzen Batterie neoliberaler Reformen. Dem PdR folgte noch im selben Jahr das umfangreiche, nach dem französischen Finanzminister benannte Reformpaket Loi Macron. Das mithilfe der Notverordnung 49-3 gegen den Widerstand der Gewerkschaften und den linken Flügel der Sozialistischen Partei beschlossene Reformpaket hatte die Abschaffung der Zugangsbeschränkung für bestimmte Berufe (Notare, Taxifahrer etc.), die Ausweitung der Nacht- und Sonntagsarbeit sowie eine umfassende Lockerung des Kündigungsschutzes zur Folge. Mit der autoritären Durchsetzung der Loi Macron reagierte die sozialistische Regierung auf die Forderungen der EUKommission und den steigenden Druck aus Deutschland (Syrovatka 2016). So sollte die Umsetzung der Loi Macron nach Aussage von Emanuel Macron in erster 
Linie ein „Reformsignal an die europäischen Partner und vor allem Deutschland" sein (Le Monde, 16.10.2014).

An die Loi Macron schließt nun der aktuelle Gesetzesentwurf für die Reform des Arbeitsrechts, die sogenannte Loi El Khomri - benannt nach der Arbeitsministerin Myriam El Khomri - an. Der Entwurf enthält im Kern keine wirklich neue Idee, knüpfen die Vorschläge doch an die Arbeitsrechtsreformen der letzten Jahrzehnte an und weisen damit eine stärkere Kontinuität auf, als die Verfasserin und ihre KritikerInnen zugeben würden: So sollen der Kündigungsschutz gelockert, betriebsbedingte Entlassungen erleichtert und die betriebliche Ebene für Vereinbarungen der Sozialpartner gestärkt werden. Jedoch geht der Gesetzesentwurf deutlich über die angebotspolitische Stoßrichtung der bisheriger Regierungen hinaus:

Lockerung der Überstundenregelung: Durch Betriebsvereinbarungen soll die Wochenarbeitszeit für den Zeitraum von 16 Wochen auf 48 Stunden (in Ausnahmefällen sogar auf 60 Stunden) erhöht und Arbeitszeiten von bis zu 12 Stunden am Tag angeordnet werden können. Auch soll die gesetzliche Vergütungsregelung von Überstunden durch Betriebsvereinbarungen abgeschwächt werden können.

Lockerungdes Kündigungsschutzes: Die Definition von „betriebsbedingten Kündigungen" soll stark erweitert werden, sodass schon kleinste Verluste Gründe für Kündigungen darstellen können.

Stärkung von Vereinbarungen auf der betrieblichen Ebene: Durch „Entwicklungsvereinbarungen" zwischen Beschäftigten und Arbeitgebern soll über einen Zeitraum von maximal zwei Jahren durch höhere Arbeitszeiten und Lohnverzicht die wirtschaftliche Expansion des Betriebes ermöglicht werden. Die Vereinbarungen unterliegen der Zustimmung durch die ArbeitnehmerInnen, jedoch soll der Arbeitgeber bei Nichtzustimmung Entlassungen aus wirtschaftlichen Gründen vornehmen können.

Schwächung von Gewerkschaften: Betriebsinterne Referenden zu Fragen wie der Wochenarbeitszeit oder zur Gehaltsgestaltung sollen bindend werden, womit Blockaden einzelner Gewerkschaften bei Betriebsentscheidungen umgangen werden können.

Schwächung der Arbeitsgerichte ${ }^{2}$ : Die Höhe von Abfindungen soll gesetzlich begrenzt werden. Der Entwurf sieht dabei relativ niedrige Obergrenzen vor: Nach zwei Jahren Betriebszugehörigkeit soll die Abfindung nur drei Monatsgehälter betragen dürfen, nach 20 Jahren und mehr sogar nur maximal 15 Monatsgehälter.

Die Arbeitsrechtsreform wird das letzte große Gesetz der Regierung Valls vor den Präsidentschaftswahlen im Mai 2017 werden. Umso stärker liegt der Druck auf der Regierung, die nun vorgelegte Reform des Arbeitsrechtes ohne größere Änderungen durchzusetzen, vor allem nach der gescheiterten Verfassungsänderung zur Staatsbürgerschaft. Zwar hatte Hollande zu Beginn seiner Amtszeit angekündigt, sich an den Arbeitslosenzahlen messen zu lassen, jedoch finden sich die Beweggründe für die Arbeitsrechtsreform vor allem im verstärkten Druck verschiedener Kapitalverbände und der EU. Schon seit 2014 fordert die

2 Schon die Loi Macron sollte Abfindungen begrenzen und die Macht der Arbeitsgerichte beschneiden, scheiterte jedoch am Verfassungsrat. Die Loi El Khomri stellt nun den zweiten Versuch dar. Während des Schreibprozesses dieses Artikels zog die Regierung Valls als Folge des Protests der reformorientierten Gewerkschaften die Festsetzung von Obergrenzen für Abfindungen zurück. 
EU-Kommission im Rahmen des Europäischen Semesters eine umfassende Reform des Arbeitsrechts und eine Lockerung der Arbeitszeit- und Kündigungsregelungen. Durch die erneute Ankündigung der Regierung Valls, auch das Defizitziel für 2017 zu verfehlen, steht Frankreich nun unter erhöhtem Druck, den Forderungen nach „strukturellen Reformen“ nachzukommen (Syrovatka 2016).

Getragen wird der Reformkurs seit der neoliberalen Wende wesentlich von den transnationalen und exportorientierten Kapitalfraktionen, die in Frankreich durch den Gesamtunternehmensverband $M E D E F$ und die Vereinigung der französischen Großunternehmen afep repräsentiert werden. Beide Verbände hatten in der Vergangenheit immer wieder Einfluss auf die Reformpolitik der sozialistischen Regierung genommen und seit Jahren Druck ausgeübt, das Arbeitsrecht zu reformieren. Daher wurde der Gesetzentwurf für die Loi El Khomri begrüßt und Unterstützung signalisiert, jedoch kritisiert, dass die Regierung durch die weitere Vergütungsverpflichtung von Überstunden an der 35-Stunden-Woche festhalte. Zudem fürchten die Unternehmensverbände eine Abschwächung des Entwurfs in der $\mathrm{Na}$ tionalversammlung. Unterstützt wird der Reformkurs überdies von den EU- Institutionen, welche in ihren länderspezifischen Empfehlungen eine Reform des französischen Arbeitsrechts gefordert hatten. Der externe Druck durch die EU hatte die Handlungs- und Kompromissspielräume der Regierung schon in der Vergangenheit verengt, was sich zuletzt in der autoritären Durchsetzung der Loi Macron manifestierte (Syrovatka 2016). Bei der vorliegenden Loi El Khomri scheint dies ebenso der Fall zu sein, kündigte Premierminister Valls doch schon im Februar 2016 an, die Reform des Arbeitsrechts, gleichzeitig zur
Loi Macron, mithilfe des Notparagrafen 49-3 auch gegen den Widerstand des Parlaments und der eigenen Partei durchzusetzen. Dieser Paragraf sieht die Annahme eines Gesetzes ohne die Abstimmung der Nationalversammlung vor, ist jedoch an ein Misstrauensvotum gegen die Regierung gekoppelt (\$49-2). ${ }^{3}$ Seit der Reform 2008 darf er nur einmal pro Sitzungsperiode eingesetzt werden, wenn die Gesetzesvorlage nicht den Staatshaushalt oder die Finanzierung der sozialen Sicherheit betrifft.

Scharfe Kritik kommt dagegen von Teilen der Gewerkschaften und breiten Teilen der Zivilgesellschaft. Nach Umfragen des Meinungsforschungsinstituts ODEXA lehnen mehr als 70 Prozent der französischen Bevölkerung eine Reform des Arbeitsrechts ab. Unter den 18- bis 34-Jährigen ist die Ablehnung mit 78 Prozent am größten. Sogar der liberale Wirtschaftswissenschaftler Jacques Attali kritisierte gegenüber dem TV-Sender BMFTV: „Man sieht in dem Reformvorschlag nur, was die Arbeitgeber gewinnen können. Man könnte glauben, die Kopie eines MEDEF-Dokuments in der Hand zu halten“. Auch der linke Flügel der Parti Socialiste kritisierte die Arbeitsrechtsreform als Angriff auf die sozialen Rechte.

Die Gewerkschaften sind gespalten. Während die linken Gewerkschaften CGT, FO und SUD einen Dialog mit

3 Sollte die Regierung den Paragrafen 49-3 anwenden, so wird in dessen Folge eine Misstrauensabstimmung in der Assemblée nationale angesetzt. Wenn das Parlament der Regierung in dieser Abstimmung weiterhin ihr Vertrauen ausspricht, so gilt das Gesetz auch ohne Abstimmung als angenommen. Scheitert die Regierung mit der Vertrauensfrage, so steht es dem Präsidenten frei, das Parlament aufzulösen. 
der Regierung ablehnen und die sofortige Rücknahme des Gesetzentwurfs fordern, verfolgen die reformistischen Gewerkschaften CFDT, CFE-CGC und UNSA eine Strategie der sozialen Partnerschaft. Im Dialog mit den Unternehmensverbänden und der Regierung soll der Gesetzentwurf in vielen Punkten abgeschwächt werden, denn auch die reformistischen Gewerkschaften bewerten den Entwurfals „einseitig und ungerecht". Dafür haben sie 745 Änderungsanträge eingebracht, wobei davon auszugehen ist, dass die Regierung in wesentlichen Punkten auf diese eingehen wird. Zusammen mit den Unternehmerverbänden und dem Mehrheitsflügel in der Sozialistischen Partei bildeten die reformorientierten Gewerkschaften in der Vergangenheit einen wettbewerbskorporatistischen Block, der die Umsetzung neoliberaler Projekte unterstützte. Gleichzeitig führte die Inkorporierung der CFDT sowie die große Schwäche der gesellschaftlichen Linken dazu, dass sich gegen die neoliberale Arbeitsmarktpolitik kaum Protest regte. Während gegen die Rentenreform 2010 noch mehrere Millionen Menschen auf die Straße gingen und sich selbst 2012 im Zuge der Präsidentschaftswahlen noch landesweit rund 750.000 Menschen an den Demonstrationen zum 1. Mai gegen Nicolas Sarkozy beteiligten, gelang es seit dem Amtsantritt von Hollande landesweit kaum mehr, 300.000 Menschen zu mobilisieren.

Anders als die reformorientierten Gewerkschaften verfolgen die linken Gewerkschaften eher eine konfrontative Strategie gegenüber der Regierung und versuchen, durch Protest auf der Straße und Streiks in den Betrieben, die Regierung zum Einlenken zu bewegen. Damit blieben sie in der jüngeren Vergangenheit jedoch ziemlich erfolglos, auch weil sich die größte linke Gewerkschaft CGT nach internen
Grabenkämpfen infolge eines Finanzskandals des ehemaligen Vorsitzenden Thierry Lepaon in einem desolaten Zustand befindet. Deshalb war es überraschend, dass dem Aufruf der linken Gewerkschaften am 9. März 2016 landesweit rund 500.000 Menschen und wenige Wochen später, am 31. März, sogar mehr als eine Million folgten. Die Demonstrationen wurden dabei nicht nur durch die traditionellen Streiks bei der Bahn (SNCF) und im Pariser Nahverkehr (RATP), sondern ebenso durch Blockaden von mehr als 500 Schulen und 200 Universitäten begleitet, was kein neues Phänomen ist, jedoch in ihrer Massivität ein neues Niveau erreicht. Denn neben den Gewerkschaften hatten zudem SchülerInnen- und Studierendenorganisationen zu den Demonstrationen aufgerufen, die als Träger des zivilgesellschaftlichen Protestes auch außerhalb des gewerkschaftlichen Aktionsrahmens gegen die Arbeitsrechtsreform demonstrierten.

Schon vor der Demonstration am 31. März waren es vor allem junge Menschen, die ihren Unmut über die Arbeitsmarktreform v.a. im Internet mit Hilfe von Onlinepetitionen und dem Hashtag \#OnVautMieuxQueÇa Luft machten. Und sie waren es, die nach den Demonstrationen auf der Place de la République blieben und damit die Bewegung Nuit Debout anstießen. Zwar war die Platzbesetzung in Paris im Vorfeld vom linken Kollektiv Convergence des luttes um den Filmemacher François Ruffin organisiert worden, jedoch konnte sich die Dynamik von Nuit Debout erst durch die breite Unterstützung von Studierenden, SchülerInnen und Intellektuellen entfalten. Damit weist die Bewegung große Ähnlichkeiten mit der spanischen 15M-Bewegung auf, waren deren Träger doch ebenfalls hauptsächlich junge weiße AkademikerInnen. Doch es gibt noch weitere Ähnlichkeiten 
zwischen den beiden Bewegungen: Auch Nuit Debout ist basisdemokratisch und horizontal organisiert sowie stark auf deliberative Entscheidungen fokussiert. Es gibt keine Hierarchien, jede und jeder kann bei allem mitreden und Diskussionen einfordern. Der Austausch über Visionen und Utopien steht im Vordergrund, diese werden in den verschiedenen Plena und Arbeitsgemeinschaften genauso diskutiert wie gemeinsame Aktionen und Forderungen. Hinzu kommt, dass fast die komplette Außen- und Innenkommunikation sowie die eigene Mobilisierung über das Internet stattfinden. Die komplette Organisationsstruktur ist offen und kann eingesehen werden, die Plena werden live übertragen. Ebenso wie die 15M-Bewegung will sich Nuit Debout offiziell nicht in ein politisches Lager einordnen lassen, obwohl ihre Nähe zur gesellschaftlichen Linken kaum zu übersehen ist. Doch lehnt sie die alten politischen Kategorien ebenso ab wie Parteien und versteht sich vielmehr als Kristallisationspunkt verschiedener Kämpfe und Forderungen. Ihre politischen Diskussionen sind daher gleichsam nicht nur auf die Rücknahme der Arbeitsrechtsreformen begrenzt, sondern umfassen ebenso die EU-Austeritäts- und Migrationspolitik sowie antisexistische, (queer-)feministische, antirassistische und andere Thematiken. Eine politische Positionsbestimmung ist daher kaum möglich und findet hauptsächlich in den beteiligten linken und studentischen Organisationen statt. Jedoch existiert ein gewisser antifaschistischer und antirassistischer Grundkonsens, welcher die Solidaritätsarbeit für Geflüchtete in mehreren Städten ebenso miteinschließt wie Platzverweise für AnhängerInnen des Front National.

Auch wenn die Pluralität politischer Positionen auf den ersten Blick diffus erscheint, so spiegelt sich in ihnen die
Begründung für die Existenz von Nuit Debout wider: Die Regierung Valls hat durch ihre Sicherheitspolitik (Ausnahmezustand), ihre restriktive Asylpolitik sowie ihre neoliberale Arbeitsmarktpolitik die Grenze des Ertragbaren für viele Menschen überschritten. Die Arbeitsrechtsreform war daher nur der Tropfen, der das Fass zum Überlaufen brachte.

Die Proteste gegen die Loi El Khomri sind die größten seit Hollandes Amtsantritt, jedoch konnten sie bisher nicht an die Proteste gegen die Rentenreform 2010 heranreichen. Grund dafür sind vor allem die reformorientierten Gewerkschaften, die anders als 2010 nicht zu den Protesten mobilisiert haben. Dies wäre jedoch notwendig, um die Arbeitsrechtsreformen verhindern zu können, da nur zusammen mit der CFDT eine landesweite (Streik-) Bewegung möglich wäre, die den nötigen Druck auf die Regierung entwickeln könnte. Eine strategische Umorientierung der reformorientierten Gewerkschaften gilt jedoch nahezu als unmöglich, sind diese doch personell wie strukturell tief mit der sozialistischen Regierung verwoben. Eine Konfrontation gegenüber den staatlichen Institutionen und Akteuren könnte den sozialen Dialog als Verhandlungsarena beschädigen, was für die reformorientierten Gewerkschaften einen starken Verlust der eigenen institutionellen Macht bedeuten würde.

Doch selbst wenn die Arbeitsrechtsreform durch die derzeitige Bewegung nicht verhindert werden kann, so wird sie Frankreich dennoch verändern. Vor allem die Platzbesetzungen zeigen, dass das politische System der V. Republik in einer tiefen Krise ist, sind sie doch ein weiterer Ausdruck des großen Zwiespalts zwischen der Bevölkerung und den sich selbst reproduzierenden politischen und ökonomischen Eliten (Syrovatka 2015: 
390). Die Krise des politischen Systems ist dabei kein neues Phänomen, jedoch konnte die Linke bisher kaum einen Nutzen daraus ziehen. Allein deshalb hat die Bewegung das Potenzial, allen Spaltungsversuchen der Regierung zu widerstehen, zur Revitalisierung der gesellschaftlichen Linken beizutragen und über die Thematik der Arbeitsmarktpolitik zu einem politischen Akteur heranzuwachsen. Zwar sind die Berührungsängste und Vorurteile zwischen der Bewegung und den institutionellen Akteuren wie Gewerkschaften und linken Parteien noch relativ hoch, jedoch existiert bereits jetzt eine hohe Unterstützung der v.a. studentischen Bewegung bis hinein in den linken Flügel der Sozialistischen Partei.

Nichtsdestotrotz bestehen zwischen den beiden Hauptakteuren der Proteste, den linken Gewerkschaften und der NuitDebout-Bewegung, hohe habituelle und inhaltliche Grenzen. Beide Seiten hegen teilweise große Vorbehalte gegenüber einander. Eine bessere Zusammenarbeit zwischen den beiden Hauptakteuren der Bewegung könnte zu einer stärkeren Mobilisierung führen und zugleich unterschiedliche Milieus ansprechen. Denn bisher scheint es nicht so, dass die Bewegung über den Kreis der Studierenden und der gesellschaftlichen Linken hinaus mobilisierungsfähig ist. Auch das Aufkommen der Platzbesetzungen hat nicht dazu geführt, dass sich etwa Prekarisierte oder unorganisierte ArbeiterInnen vermehrt an den Protesten beteiligen. Zwar existieren derzeit Ansätze, dieser „unsichtbaren Spaltung" durch eine Mobilisierung in die Banlieues entgegenzuwirken, jedoch blieben diese bisher erfolglos. Die Öffnung gegenüber anderen gesellschaftlichen Milieus ist jedoch für die Aufrechterhaltung der Bewegung ebenso wichtig wie die Zuspitzung der sozialen Frage in der politischen Kommunikation. Letztendlich wird der Erfolg der Bewegung mittelfristig davon abhängen.

\section{Literatur}

Boltanski, Luc/Chiapello, Ève (2006): Der neue Geist des Kapitalismus, Konstanz.

Delbar, Catherine/Léonard, Evelyne (2002): Le travail intérimaire. In: Courrier hebdomadaire du CRISP 33(1778): 5-45.

Lux, Julia (2015): France in limbo. In: French Politics 13(1): 84-102.

Syrovatka, Felix (2015): Der Aufstieg der Madame Le Pen. In: PROKLA 45(3): 387-409.

- (2016): Die Reformpolitik Frankreichs in der Krise, Wiesbaden i.E.

Vail, Mark I. (2010): Recasting Welfare Capitalism, Philadelphia. 